() 2017 IEEE. Personal use of this material is permitted. Permission from IEEE must be obtained for all other uses, in any current or future media, including reprinting/republishing this material for advertising or promotional purposes, creating new collective works, for resale or redistribution to servers or lists, or reuse of any copyrighted component of this work in other works. 


\title{
A Dual Patch Polarization Rotation Reflective Surface and Its Application to Ultra-Wideband RCS Reduction
}

\author{
Yongtao Jia, Ying Liu, Member, IEEE, Y. Jay Guo, Fellow, IEEE, Kun Li, and Shuxi Gong
}

\begin{abstract}
An ultra-wideband polarization rotation reflective surface (PRRS) with a high polarization conversion ratio (PCR) is proposed, which can reflect the linearly polarized incident wave with $90^{\circ}$ polarization rotation. The unit cell of the proposed PRRS consists of a square and an $L$-shaped patches printed on a substrate, which is covered by a superstrate and backed by a metallic ground. Each of the two patches is connected to the ground using two metallic vias, respectively. Compared with the previously reported PRRS, the polarization rotation bandwidth of the proposed PRRS is enhanced from $49 \%$ to $97 \%$ with a high PCR of $96 \%$. Moreover, the frequency responses within the operation frequency band are consistent under oblique incident waves. Furthermore, the designed PRRS is applied to ultra-wideband radar cross section (RCS) reduction by forming a checkerboard surface. The numerical relationship between RCS reduction of the checkerboard surface and the PCR of the PRRS is discussed. A 10-dB RCS reduction is achieved over an ultra-wideband of $103 \%$. To validate the simulation results, prototypes of the checkerboard surface is fabricated and measured. Good agreement between the experiments and simulations has been obtained.
\end{abstract}

Index Terms-Polarization rotation, ultra-wideband, reflective surface, radar cross section (RCS)

\section{I.INTRODUCTION}

Polarization rotators are a type of import electromagnetic structures which have been intensively investigated in the microwave and quasi-optical frequencies in the past few years [1-5]. Generally speaking, polarization rotators can be categorized into transmission and reflection types. For the transmission type, one can exploit either the birefringence effect of anisotropic metamaterials [6-8] or the optical activity of chiral metamaterials [9-11]. Most of these structures operate at a narrow single or two separated narrow bands $[6-9,11]$. In [10], an ultra-thin chiral metamaterial slab stacked with twisted complementary split-ring resonators for highly efficient broadband polarization transformation is proposed. Its

This work is supported by the National Natural Science Foundation of China (No. 61372001).

Yongtao Jia, Ying Liu and Shuxi Gong are with the Science and Technology on Antenna and Microwave Laboratory, Collaborative Innovation Center of Information Sensing and Understanding, Xidian University, Xi'an 710071, China (e-mail: liuying@mail.xidian.edu.cn;).

Y. J. Guo is with the Global Big Data Technologies Center, University of Technology Sydney (UTS), Sydney, Australia (e-mail: jay.guo@uts.edu.au). conversion efficiency reaches up to $96 \%$ with a bandwidth of $24 \%$. For the reflection type, the plasmon resonances and asymmetric high impedance surface are utilized to realize the polarization rotation in [12-19]. The reflection type polarization rotators proposed in the literature either work at a few separated narrow polarization rotation frequency bands or don't have high enough PCRs over the polarization rotation band.

In [20], a broadband PRRS is proposed by connecting the square patch to the ground using two asymmetric vias. A $49 \%$ bandwidth is obtained with a high PCR of $96 \%$. Besides, another ultra-wideband PRRS with a periodic array of quasi L-shaped patches is proposed as well, which increases the polarization rotation bandwidth further to $103 \%$. However, the PCR of the ultra-wideband PRRS is only 50\% in some frequency range. In this paper, a novel ultra-wideband and high-efficiency PRRS is presented by employing single layer dual patch unit cells. The unit cell consists of a square and an L-shaped patches printed on a substrate, which is covered by a superstarte and a backed by a metallic ground. Unit cells employing square and L-shaped patches individually have been reported in [20]. When they are combined to form a dual patch unit and covered by a superstrate, however, the electrical size of the unit cell is reduced while the operation bandwidth is broadened by $\mathrm{xx} \%$ with almost the same PCR. Furthermore, we apply the dual patch based PRRS to a metallic sheet to reduce the RCS an ultra-wideband. By arranging the unit cells of the PRRS in four orthogonal directions, a checkerboard surface with low RCS is formed and a 10-dB RCS reduction is achieved in an ultra-wideband of $103 \%$. In the literature, it has been reported that, by combining the AMC and PEC or two different kinds of AMC with $180^{\circ}$ phase difference in a checkerboard configuration, the two reflections from the two surfaces cancel each other under a normal incident plane wave and re-radiate the energy in other directions, thus reducing the RCS in the normal direction [21-24]. The former method can only reduce RCS in a narrow band while the latter one requires two kinds of $\mathrm{AMC}$ with $180^{\circ}$ phase difference over a wide frequency band to realize wideband RCS reduction. In contrast, an ultra-wideband RCS reduction can be achieved by using only one kind of PRRS with the proposed configuration, thus simplifying the design and fabrications process.

The paper is organized as follows. Section II describes the topological structure of the ultra-wideband dual-patch PRRS with a high PCR and presents its design procedure. Then, 
Section III presents a checkerboard surface formed by the proposed PRRS for ultra-wideband RCS reduction and discusses the relationship between the RCS reduction and the PCR of the PRRS. Finally, the paper is concluded in Section IV.

\section{II.NOVEL ULTRA-WIDEBAND PRRS WITH A HIGH PCR}

This section presents the design of a novel ultra-wideband PPRS with a high PCR. The unit cell of the proposed PRRS is depicted in Fig. 1. It consists of a square and an L-shaped metallic patches printed on the substrate backed by a metallic ground plane, and each of the two patches are connected to the metallic ground by two metallic vias loaded in a diagonal direction. The dual patch unit cell is covered by a superstrate. The period of the unit cell of the proposed PRRS is $6.0 \mathrm{~mm}$ and the permittivity of both the substrate and the superstrate is 2.65 $(\tan \delta=0.001)$. The other parameters are given in Table I.

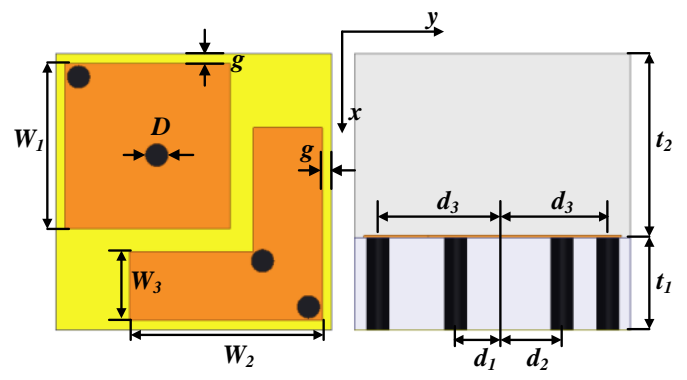

Fig.1 The topological structure of the unit cell of the ultra-wideband PRRS with a high PCR.

TABLE I

UNIT CELL DiMENSION (MM)

\begin{tabular}{|c|c|c|c|c|}
\hline $\mathrm{W}_{1}$ & $\mathrm{~W}_{2}$ & $\mathrm{~W}_{3}$ & $\mathrm{D}$ & $\mathrm{g}$ \\
\hline 3.6 & 4.2 & 1.5 & 0.5 & 0.2 \\
\hline $\mathrm{d}_{1}$ & $\mathrm{~d}_{2}$ & $\mathrm{~d}_{3}$ & $\mathrm{t}_{1}$ & $\mathrm{t}_{2}$ \\
\hline 0.8 & 1.5 & 2.5 & 2 & 4 \\
\hline
\end{tabular}

In the following, we define $r_{x y}=E_{x}^{R e f} / E_{y}^{I n c}$ and $r_{y y}=E_{y}^{R e f} /$ $E_{y}^{I n c}$ to represent the reflection ratio of y-to-x, and y-to-y polarization conversions, where the $E_{y}^{I n c}$ represents the electric field of the y-polarized incident EM wave, while the $E_{y}^{R e f}$ and $E_{x}^{R e f}$ represent the electric fields of the y- and x-polarized reflected EM waves, respectively.

To understand the response of the proposed PRRS to the incident EM wave with y-polarized electric field, we decompose the electric field of the incident $\mathrm{EM}$ wave $\mathrm{E}_{\mathrm{i}}$ into two perpendicular components, $\mathrm{E}_{\mathrm{i} u}$ and $\mathrm{E}_{\mathrm{i} v}$, as shown in Fig. 2a. When the incident EM waves are $u$-polarized and $v$-polarized, respectively, the reflected magnitudes and phases are shown in Figs. 2b. It can be seen that the magnitudes of the two curves are approximately the same. Most of the energy is reflected with the same polarization as the incident wave. Furthermore, the relative phase retardation is roughly $180^{\circ}$ from 6.2 to $17.8 \mathrm{GHz}$. Taking the frequency point where the reflected phase $\varphi_{u}$ is $0^{\circ}$, for example, we display the electric vectors of the incident and reflected waves in Fig. 2a. When $\varphi_{u}$ is $0^{\circ}, \varphi_{v}$ is $-180^{\circ}$ for the reason that the phase difference between $\varphi_{u}$ and $\varphi_{v}$ is approximately $180^{\circ}$. Thus, the reflected electric field $\mathrm{E}_{\mathrm{r} u}$ is equal to $\mathrm{E}_{\mathrm{i} u}$ while $\mathrm{E}_{\mathrm{r} v}$ is equal to $-\mathrm{E}_{\mathrm{i} v}$. Meanwhile, the magnitudes of the reflected fields are equal to those of the incident fields. Then, the total reflected electric field of the reflected $\mathrm{EM}$ wave $\mathrm{E}_{\mathrm{r}}$ can be obtained, which shares the same magnitude with $\mathrm{E}_{\mathrm{i}}$ but is parallel to the $\mathrm{x}$-axis. The same applies for the frequency range in which the phase difference is approximately $180^{\circ}$.

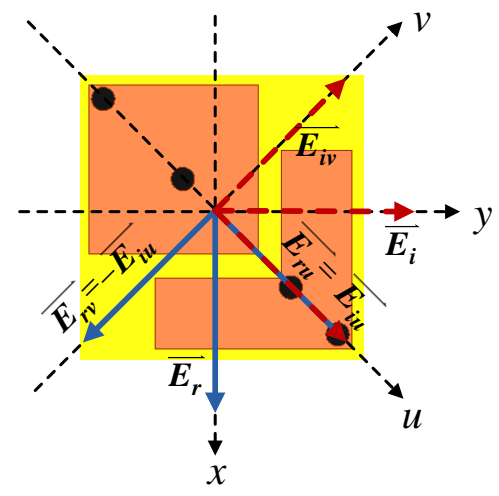

(a)

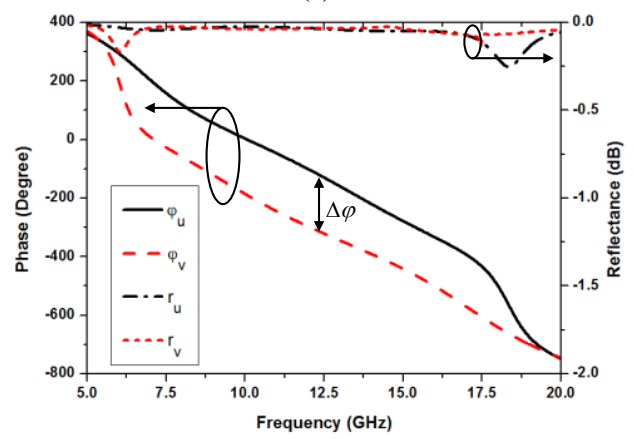

(b)

Fig. 2. (a) Intuitive scheme of y-to-x polarization conversion. (b) The reflected amplitudes and phases with the electric field of incident EM waves along $u$ - and $v$-axis, respectively.

The simulated reflection coefficients $r_{x y}$ and $r_{y y}$ of the ultra-wideband PRRS at oblique incidence of the y-polarized EM waves, are given in Fig. 3. It can be seen from Fig. 3a that with the incident angle $(\theta)$ increasing from $0^{\circ}$ to $60^{\circ}$, the $-3-\mathrm{dB}$ bandwidth of the reflection coefficient $r_{x y}$ is reduced by about $2 \mathrm{GHz}$. However, the frequency response of the proposed PRRS at lower frequency range is reasonably consistent. The reflection coefficient $r_{x y}$ is still larger than $-2 \mathrm{~dB}$ from 6 to 16 $\mathrm{GHz}$ when the incident angle increases to $60^{\circ}$. Fig. $3 \mathrm{~b}$ shows that when $\theta$ is $0^{\circ}$, there are five resonant points and the resonant points decrease to four with oblique incident EM wave. 


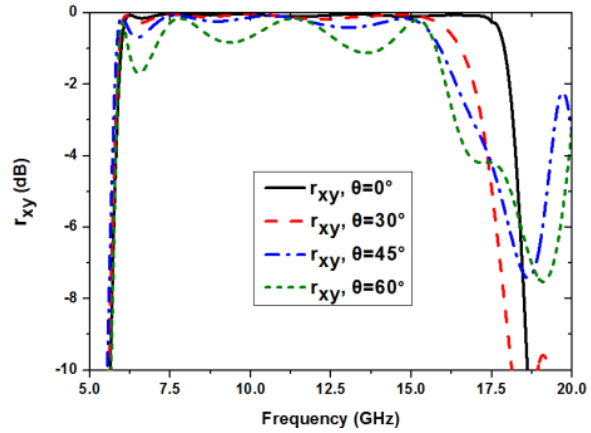

(a)

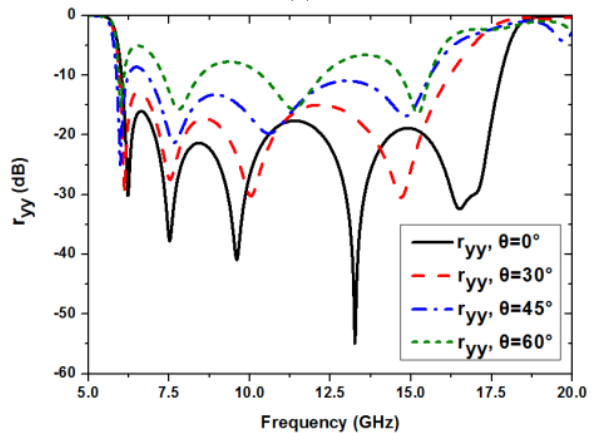

(b)

Fig. 3. Simulated reflection coefficients of the proposed PRRS at oblique incidence of EM waves: (a) $r_{x y}$ and (b) $r_{y y}$.

Here, we use the surface currents of the unit cell of the PRRS to explain of polarization rotation mechanism. When the incident EM wave is y-polarized, the $y$-direction induced current can be generated. Owing to the asymmetric structure of the PRRS, a difference of ?? (what) in the $x$-direction can be introduced by the non-uniform distribution of the surface impedance along the $x$-axis. As a result, some current will move towards the $x$-direction, which will generate an electric field $E_{x}$ along the $x$-direction. When the current moving along the $x$-direction is equal to that of the $y$-direction, the reflected field becomes orthogonal to the incident field with the same magnitude. The simulated surface current distributions on the metallic parts of the unit cell at the five polarization points (as shown in Fig. 3b) are given in Fig. 4. The intersection angle between the direction of the total surface current induced on the patches and $\mathrm{y}$-axis is $45^{\circ}$, which indicates a $90^{\circ}$ polarization rotation.
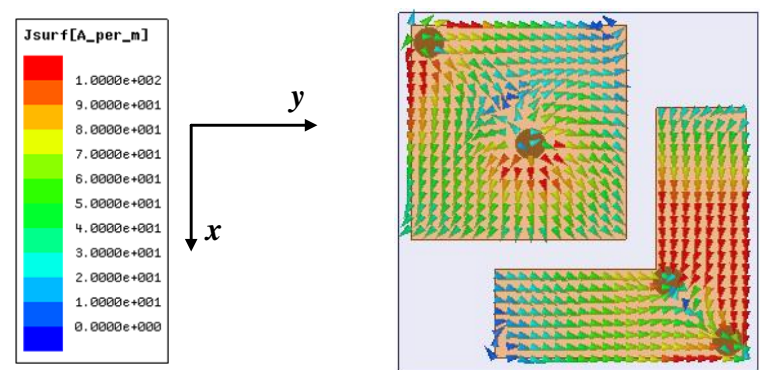

(a)

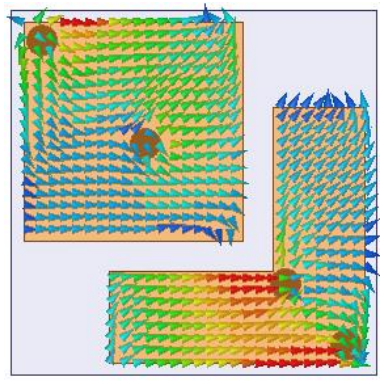

(b)

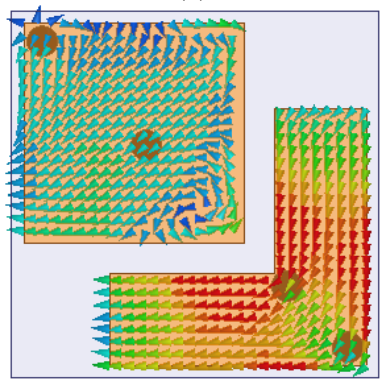

(d)

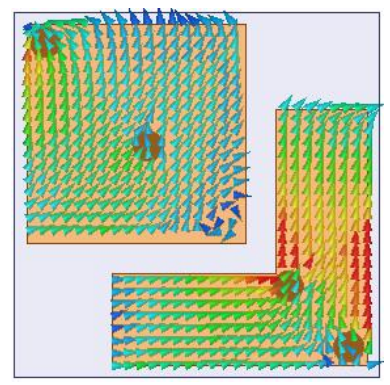

(c)

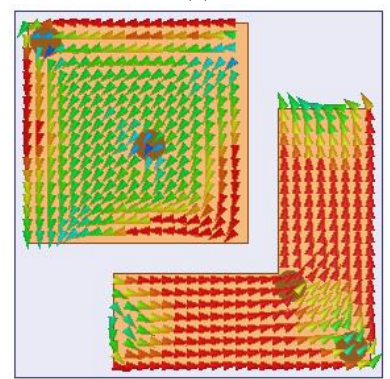

(e)
Fig. 4. The simulated surface current distributions on metallic patches of the unit cell at the five resonant points: (a) $6.42 \mathrm{GHz}$, (b) $7.68 \mathrm{GHz}$, (c) $9.38 \mathrm{GHz}$, (d) $13.3 \mathrm{GHz}$, and (d) $17.1 \mathrm{GHz}$

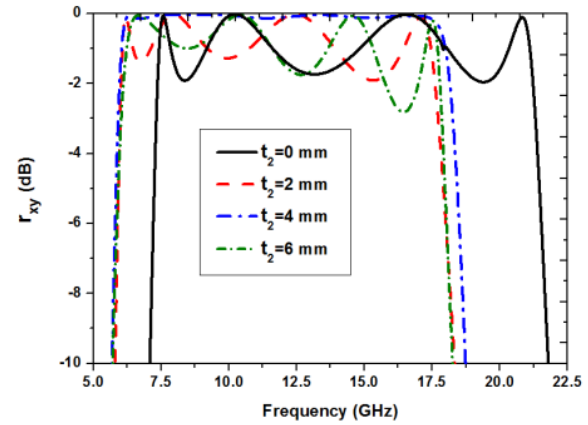

Fig.5. Simulated reflection coefficients $r_{x y}$ of the proposed PRRS for the case of different $\mathrm{t}_{2}$ increasing from $0 \mathrm{~mm}$ to $6 \mathrm{~mm}$.

The simulated reflection coefficients $r_{x y}$ of the proposed PRRS without and with different thickness of the superstrate are displayed in Fig. 5. It is shown that the thickness of the superstrate has a great influence on the reflection coefficient $r_{x y}$. When the PRRS is not covered by the superstrate, the operation band moves to the higher frequency range compared with that of the PRRS covered by the superstrate. When the thickness of the superstrate is $4 \mathrm{~mm}$, the polarization conversion efficiency of the PRRS is higher than those when the thickness is $2 \mathrm{~mm}$ and $6 \mathrm{~mm}$. With an optimized $\mathrm{r}$ thickness of the superstrate, the polarization conversion efficiency can be maximized. This is to ensure that the multiple reflections on the air-superstrate interface and the superstrate-metallic patches interface enhance the polarization conversion efficiency of the PRRS. To further study the relationship between the polarization conversion efficiency and the thickness of the superstrate, the interference theory in [25-26] can be used.

As shown in Fig. 6, the y-polarized incident EM wave on the air-superstrate interface is partially reflected back to the air and partially transmitted into the superstrate. The transmitted 
y-polarized EM wave continues to propagate in the propagation phase $\beta=\sqrt{\varepsilon} k_{0} d$ in the superstrate until it reaches the interface between the superstrate and the metallic patches. Since the metallic patches are connected to the ground sheet by the metallic vias, we treat the metallic structures and the dielectric substrate as a whole, regardless of the multiple reflection process among these structures. Thus, the transmitted EM wave is reflected on the superstrate-metallic patches interface back to the superstrate. Because of the polarization conversion characteristics of the PRRS, the reflected waves consist of both y-polarized and x-polarized components. As a result, there are two reflection coefficients, $r_{23 x y} \exp \left(i \emptyset_{23 x y}\right)$ and $r_{23 y y} \exp \left(i \emptyset_{23 y y}\right)$. Then, the partially reflection and transmission occur when the reflected EM waves reach the superstrate-air interface. After that, when the EM waves reflected from the superstrate-air interface reaches the superstrate-metallic patches interface again, there are two more reflection coefficients $r_{23 x x} \exp \left(i \emptyset_{23 x y}\right) \quad$ and $r_{23 y x} \exp \left(i \emptyset_{23 y x}\right)$. Because the proposed structure is symmetric to the diagonal, we can get that $r_{23 x y}=$ $r_{23 y x}, r_{23 x x}=r_{23 y y}, \emptyset_{23 x y}=\emptyset_{23 y x}$, and $\emptyset_{23 x x}=\emptyset_{23 y y}$.

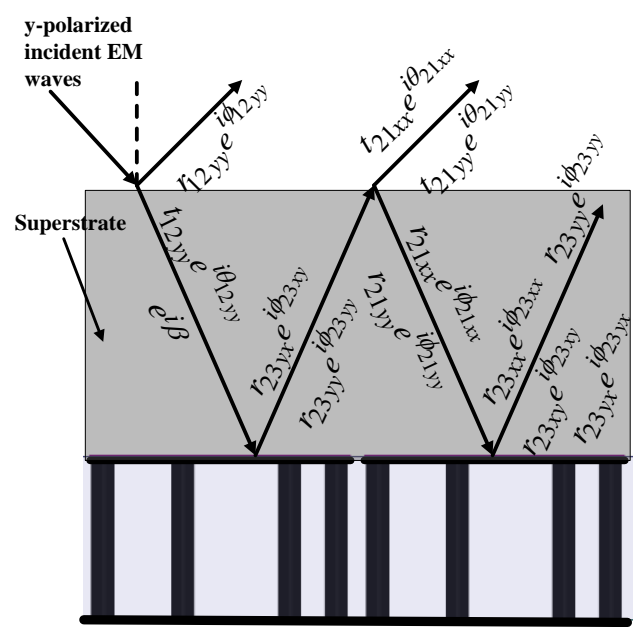

Fig. 6. The scheme of multiple reflection-transmission process described by the reflection and transmission coefficients

The magnitude and phase of the reflection coefficient at the superstrate-metallic patches are given in Fig. 7a and b, which are obtained by using the full wave EM simulation software. The reflection and transmission coefficients at the air-superstrate interface are constants since both the superstrate and air are homogeneous and isotropic. Thus, the total reflection of the x-polarized EM wave can be calculated by superposition of all multiple reflections. The PCR of the PRRS can be expressed as PCR $=\mathrm{r}_{\mathrm{xy}}{ }^{2} /\left(\mathrm{r}_{\mathrm{xy}}{ }^{2}+\mathrm{r}_{\mathrm{yy}}{ }^{2}\right)$. The calculated reflection coefficients and PCR are in good agreement with those from the simulation, respectively, as shown in Fig. 8a and b. It can be seen that the PCR is higher than $96 \%$ in a $97 \%$ bandwidth from $6.1 \mathrm{GHz}$ to $17.7 \mathrm{GHz}$.

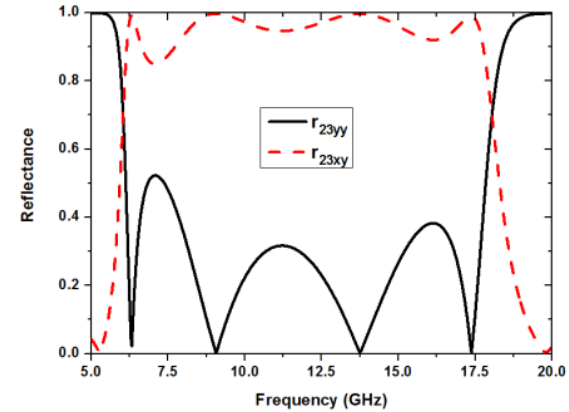

(a)

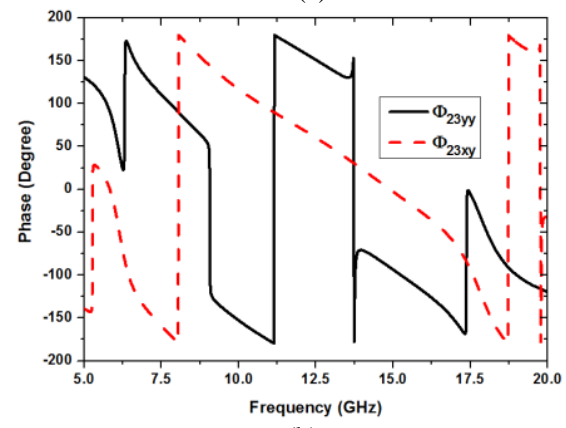

(b)

Fig.7. The reflection coefficients at the superstrate-metallic patches interface obtained by numerical simulation: (a) amplitudes and (b) phases

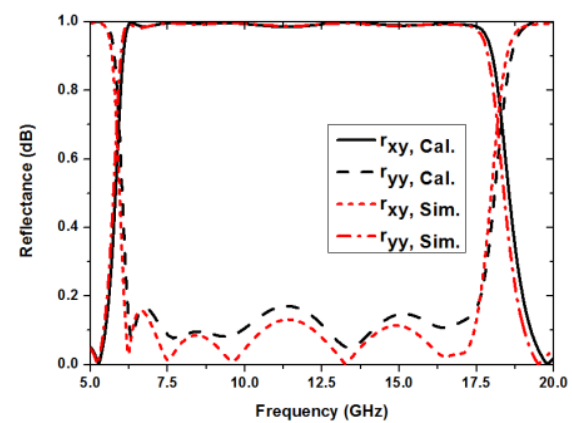

(a)

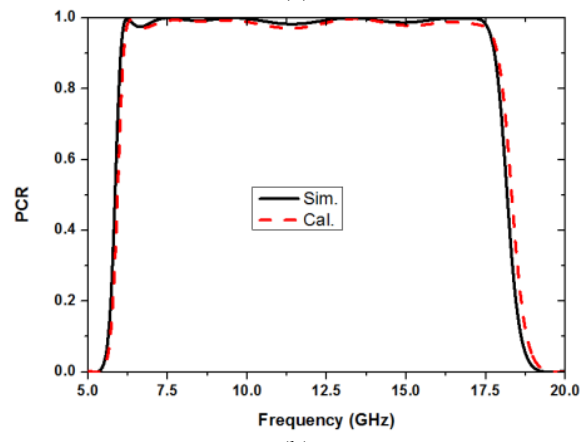

(b)

Fig.8. The calculated and simulated (a) reflection coefficients $r_{x y}$ and $r_{y y}$ and (b) PCR of the proposed PRRS.

Table II presents a comparison between the proposed PRRS and other reported polarization converters [27-29]. It shows that the proposed PRRS has an ultra-wideband in which the PCR is higher than 90\%. In deed, the PCR of the proposed PRRS is higher than $96 \%$ while the PCRs of the other reported polarization convertors undulate between $90 \% \sim 100 \%$. That's to say, not only the bandwidth but also the stability of the proposed PRRS is better than those of the other reported polarization convertors. 
TABLE II

COMPARISON WITH OTHER WIDEBAND POLARIZATION CONVERTORS

\begin{tabular}{cccc} 
& $\begin{array}{c}\text { Electrical size } \\
(\text { width } \times \text { length } \times \text { thickness })\end{array}$ & OB $(\mathrm{GHz})$ & $\mathrm{RB}(\%)$ \\
\hline Ref. [27] & $0.8 \lambda_{0} \times 0.8 \lambda_{0} \times 0.36 \lambda_{0}$ & $14.7-18$ & 20 \\
Ref. [28] & $0.35 \lambda_{0} \times 0.35 \lambda_{0} \times 0.106 \lambda_{0}$ & $10.6-17.5$ & 49 \\
Ref. [29] & $0.26 \lambda_{0} \times 0.26 \lambda_{0} \times 0.066 \lambda_{0}$ & $12.4-27.96$ & 77 \\
Present study & $0.12 \lambda_{0} \times 0.12 \lambda_{0} \times 0.12 \lambda_{0}$ & $6.03-17.78$ & 98
\end{tabular}

OB: operation bandwidth (PCR $>90 \%)$

$\mathrm{RB}$ : relative bandwidth $(\mathrm{PCR}>90)$

\section{III.APPLICATION OF THE PROPOSED PRRS ON WIDEBAND RADAR CROSS SECTION REDUCTION}

\section{A. Theoretical Analysis of the PRRS for RCS Reduction}

In this section, the PRRS proposed in Section II was used to form a checkerboard surface to reduce the RCS of metallic sheet. What's more, the numerical relationship between RCS reduction of the checkerboard surface and PCR of the PRRS is analyzed. Fig. 9 illustrates the proposed low RCS checkerboard surface. It can be seen that the checkerboard surface is obtained by arranging the unit cells of the PRRS in four orthogonal directions. Each of the four areas is formed by $16 \times 16$ unit cells with a total dimension of $96 \times 96 \mathrm{~mm}^{2}$ and the total dimension of the checkerboard surface is $192 \times 192 \mathrm{~mm}^{2}$. The RCS of a metallic ground backed substrate with the same permittivity and size as the proposed checkerboard surface is selected as the reference.

The RCS of a target $\sigma$ can be written as [30]:

$$
\sigma=4 \pi \lim _{R \rightarrow \infty} R^{2} \frac{\left|\mathrm{E}_{\mathrm{S}}\right|^{2}}{\left|\mathrm{E}_{\mathrm{i}}\right|^{2}}
$$

where the $E_{i}$ and $E_{s}$ are the electric field intensity of the incident and scattered EM waves at the target; $R$ is the distance between the target and the detection radar. According to equation (1), we can analyze the RCS of the PRRS using the electric fields of the incident and reflected EM waves at the target.

When this structure is impinged by a normal incident EM wave with a y-polarized electric field, its RCS is discussed using the reflected electric field as shown in Fig. 10. Since the proposed PRRS has the characteristics of polarization rotation, the electric field of reflected EM wave has two components: $\mathrm{X}$ polarized electric field $E_{r x}$ and y-polarized electric field $E_{r y}$. The magnitude of $\mathrm{E}_{\mathrm{rx}}$ and $\mathrm{E}_{\mathrm{ry}}$ can be expressed as

$$
\begin{aligned}
& \left|E_{r x}\right|=\left|r_{x y}\right|\left|E_{i y}\right| \\
& \left|E_{r y}\right|=\left|r_{y y}\right|\left|E_{i y}\right|
\end{aligned}
$$

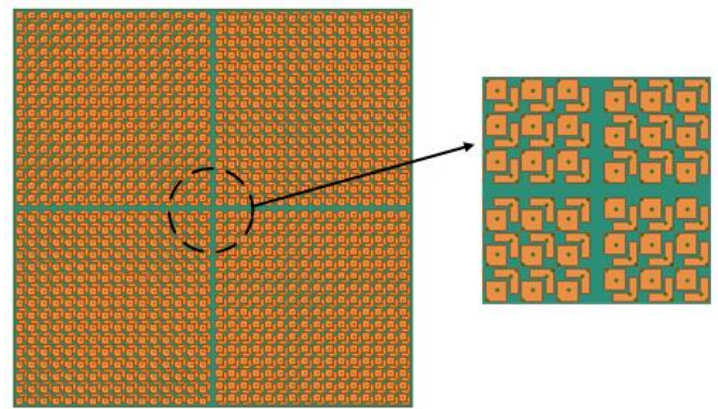

Fig. 9. Structure of the checkerboard surface formed by the proposed ultra-wideband PRRS.

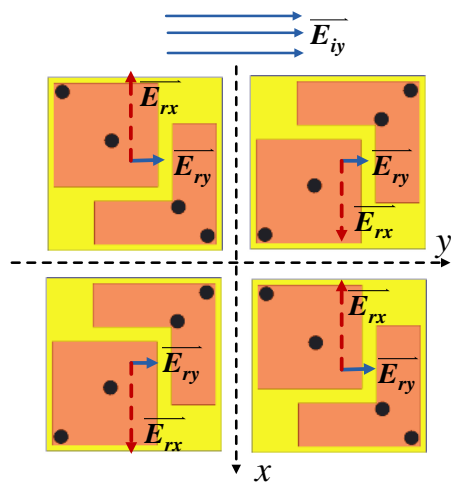

Fig. 10. Scheme of the electric field of reflected wave with y-polarized incident EM wave

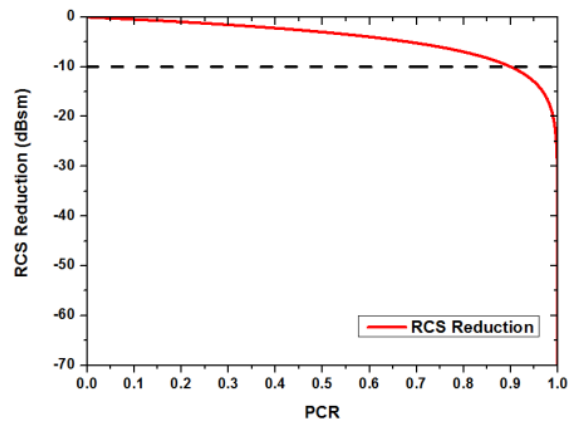

Fig. 11. The RCS reduction of the proposed checkerboard surface formed by PRRS versus PCR of the PRRS

It can be seen from Fig. 10 that the x-polarized electric fields of EM waves reflected from different parts of the checkerboard surface have a $180^{\circ}$ phase difference. Furthermore, the four orthogonal-arranged PRRSs occupy exactly the same area. Thus, the reflected EM waves with x-polarized electric field will be canceled out with each other. So the total reflected EM wave has only y-polarized electric field. Then, the RCS of the proposed checkerboard surface $\sigma_{c}$ can be written as

$$
\sigma_{c}=4 \pi \lim _{R \rightarrow \infty} R^{2} \frac{\left|\mathrm{E}_{\mathrm{ry}}\right|^{2}}{\left|\mathrm{E}_{\mathrm{iy}}\right|^{2}}
$$

The RCS reduction of the checkerboard surface, compared to that of a PEC with the same dimension, can be approximated by 


$$
\begin{aligned}
\text { RCS Reduction }(\mathrm{dB}) & =10 \log _{10}\left[\frac{\lim _{R \rightarrow \infty} 4 \pi R^{2} \frac{\left|\mathrm{E}_{\mathrm{ry}}\right|^{2}}{\left|\mathrm{E}_{\mathrm{iy}}\right|^{2}}}{\lim _{R \rightarrow \infty} 4 \pi R^{2}|1|^{2}}\right] \\
& =10 \log _{10}\left[\frac{\left|E_{r y}\right|^{2}}{\left|E_{i y}\right|^{2}}\right] \\
& =10 \log _{10}\left[\frac{\left(\left|\mathrm{r}_{\mathrm{yy}}\right|\left|\mathrm{E}_{\mathrm{iy}}\right|\right)^{2}}{\left|E_{i y}\right|^{2}}\right] \\
& =r_{y y}(\mathrm{~dB})
\end{aligned}
$$

Regardless of the dielectric loss, the numerical relationship between RCS reduction of the checkerboard and PCR of the PRRS can be represented by

$$
\begin{aligned}
\text { RCS Reduction (dB) } & =10 \log _{10}\left[\left|r_{y y}\right|^{2}\right] \\
& =10 \log _{10}\left[1-\left|r_{x y}\right|^{2}\right] \\
& =10 \log _{10}[1-P C R]
\end{aligned}
$$

By applying (6), the RCS of a checkerboard surface versus the PCR of the PRRS is displayed in Fig. 11. In the ideal case of an infinite checkerboard surface, a 10-dB RCS reduction will be obtained when the PCR of PRRS is large than $90 \%$. That's to say, in order to achieve a good RCS reduction over an ultra-wideband, an ultra-wideband PRRS with a high PCR is needed

\section{B. Results and Discussion}

The normalized monostatic RCS with respect to the reference for the proposed ultra-wideband checkerboard surface is shown in Fig. 12 to validate the above analysis. It can be seen that a 10-dB RCS reduction is achieved over more than $103 \%$ frequency bandwidth (from $5.6 \mathrm{GHz}$ to $17.7 \mathrm{GHz}$ ). What's more, the curve of RCS reduction is in agreement with that of $r_{y y}$ of the proposed PRRS, which conforms to the result of equation (5). The difference between these two curves may be due to the diffraction from the edges.

The comparison of the monostatic RCS between the checkerboard surface and the reference with oblique incident EM wave at four different frequency points are given in Fig. 13. It can be seen that the monostatic RCS of the checkerboard structure is smaller than that of the reference at most angles of the incident EM waves.

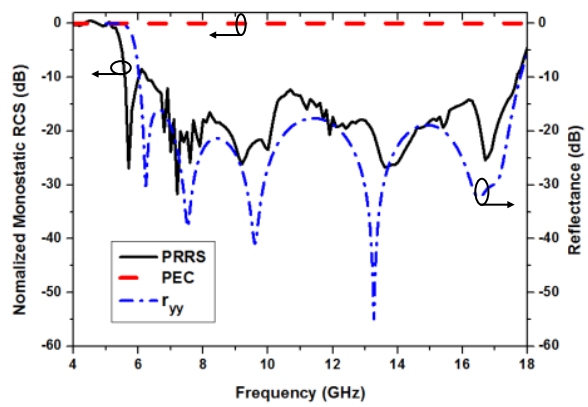

Fig. 12. Normalized monostatic RCS with respect to the reference for the proposed ultra-wideband checkerboard surface and $\mathrm{r}_{\mathrm{yy}}$ of the PRRS.

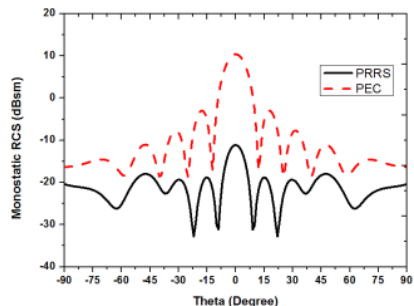

(a) $7 \mathrm{GHz}$

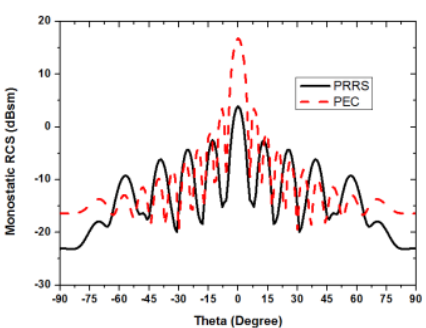

(c) $15 \mathrm{GHz}$

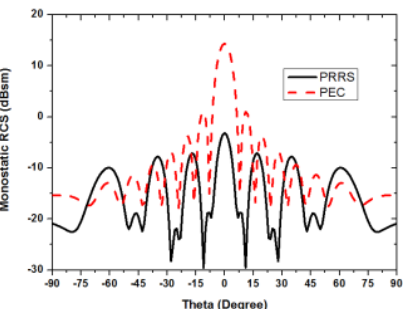

(b) $11 \mathrm{GHz}$

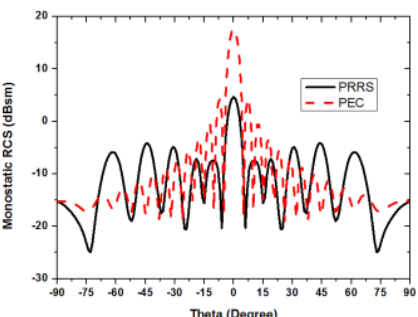

(d) $17 \mathrm{GHz}$
Fig. 13. Monostatic RCS of the checkerboard surface and the reference for oblique incident EM wave at (a) $7 \mathrm{GHz}$, (b) $11 \mathrm{GHz}$, (c) $15 \mathrm{GHz}$, and (d) $17 \mathrm{GHz}$

As we know, the energy is conserved. For the cases of RCS reduction using non-absorptive materials, the total scattered energy is not reduced. As a result, the reduction of RCS in a certain angular domain inevitably leads to the increase of the RCS in certain other angular domains. In order to investigate the distribution of the scattered energy, we compare the bistatic RCS of checkerboard surface with that of the reference at $12 \mathrm{GHz}$ for normally incident EM wave, as shown in Fig. 14. Compared with the bistatic RCS of the reference, it can be seen that the main lobe of the bistatic RCS of checkerboard surface is reduced significantly while four side lobes arise. This comparison clearly indicates where the energy reduced in the backward direction travels. To further investigate the bistatic RCS reduction of the proposed checkerboard surface, the figure of merit for bistatic reduction (FMB) is given in Fig. 15, which is first defined in [34]

$$
\operatorname{FMB}(f)=\frac{\operatorname{Max}\{\text { Bistatic } \mathrm{RCS} \text { of the proposed structure }\}}{\operatorname{Max}\{\text { Bistatic RCS of the reference }\}}
$$

where the $\operatorname{Max}\{\cdot\}$ function domain covers all bistatic scattering angles $\left(\theta_{s}, \emptyset_{s}\right)\left(0 \leq \theta_{s} \leq 90^{\circ}\right.$ and $\left.0 \leq \emptyset_{s} \leq 360^{\circ}\right)$ for a given frequency $f$ and incident angle $\left(\theta_{i}, \emptyset_{i}\right)$.

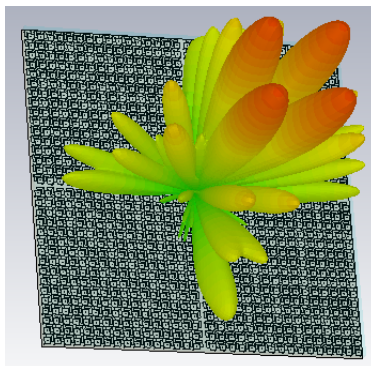

(a)

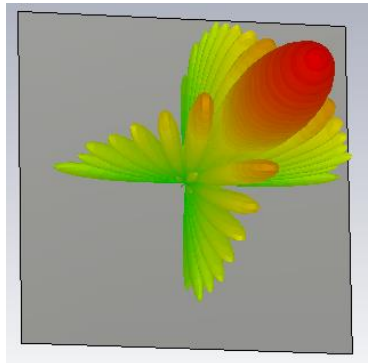

(b)
Fig. 14. Bistatic RCS at $12 \mathrm{GHz}$ for normally incident EM wave: (a) checkerboard surface and (b) reference 


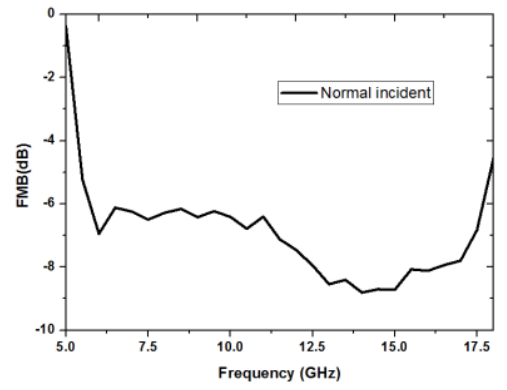

(a)

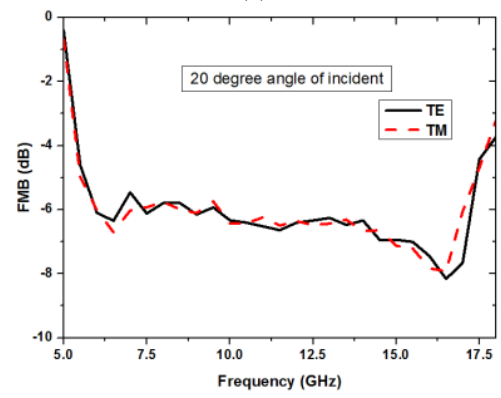

(b)

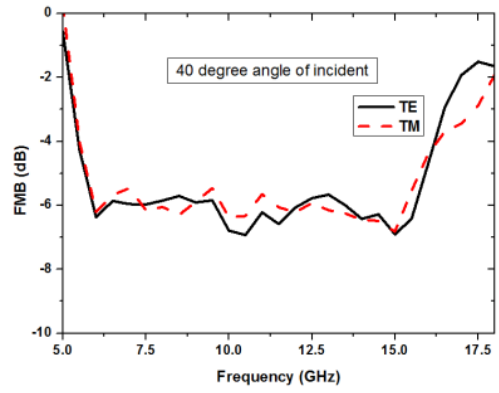

(c)

Fig. 15 Figure of merit bistatic RCS reduction (FMB) of the checkerboard surafce versus frequency at different angles of incidence

The FMB for both TE- and TM-polarized incident waves is calculated in a range of incident angles from normal to $40^{\circ}$ off normal incidence. As illustrated in Fig. 15a, a 6-dB RCS reduction bandwidth of $95 \%$ is obtained for normal incidence. For the case of oblique incidence, the amount and bandwidth of RCS reduction are decreased. A 5.5-dB RCS reduction or higher is obtained with a bandwidth of $88 \%$ up to $40^{\circ}$ angle of incidence, as shown in Fig. 15b and c.

Table III presents a comparison between the proposed checkerboard surface and other reported low RCS structures [23, 31-34]. It shows that the proposed PRRS has an ultra-wide frequency band in which the Monostatic RCS reduction is larger than $10 \mathrm{~dB}$ and the FMB is less than $-6 \mathrm{~dB}$. The comparison implies the good performance of the proposed low RCS surface for both monostatic and bistatic RCS reduction

TABLE III

COMPARISON WITH OTHER METHODS FOR WIDEBAND RCS REDUCTION

\begin{tabular}{ccc}
\hline & $\begin{array}{c}\text { Bandwidth (-10dB } \\
\text { RCS reduction) }\end{array}$ & $\begin{array}{c}\text { Bandwidth } \\
(\mathrm{FMB}<-6 \mathrm{~dB})\end{array}$ \\
\hline Ref[23] & $40 \%$ & - \\
Ref[31] & $60 \%$ & - \\
Ref[32] & $67 \%$ & - \\
\hline
\end{tabular}

\begin{tabular}{ccc}
\hline Ref[33] & $74 \%$ & - \\
Ref[34] & $13 \%$ & $66 \%$ \\
Present Study & $103 \%$ & $95 \%$ \\
\hline
\end{tabular}

The checkerboard surface is fabricated as shown in Fig. 16. Its scattering property is measured in a microwave anechoic chamber. Displayed in Fig. 17 is the schematic view of the experimental setup. Three pairs of horn antennas, selected as emitters and receivers, have been utilized to cover the frequency band from $5.85 \mathrm{GHz}$ to $18 \mathrm{GHz}$. These correspond with the following bands: $5.85 \sim 8.2 \mathrm{GHz}, 8.2 \sim 12.4 \mathrm{GHz}$ and 12.4 18 GHz. Fig. 18 Shows the Comparison between the simulated and measured RCS of the checkerboard structure. It can be seen that a reasonably agreement between the measured and simulated results is obtained in the designed frequency band.

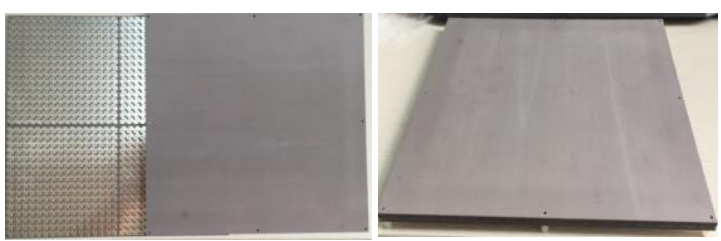

Fig. 16. Photograph of the proposed checkerboard surface

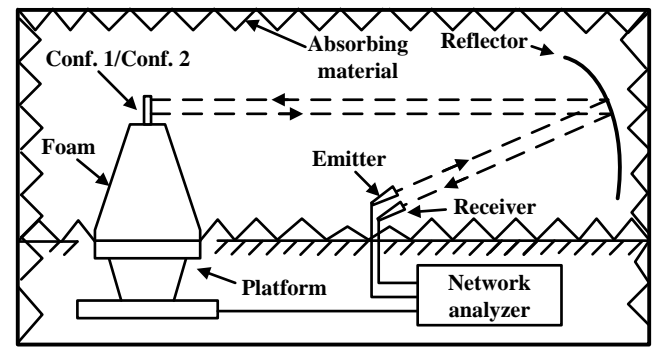

Fig. 17. Schematic view of the RCS measurement setup in an anechoic chamber

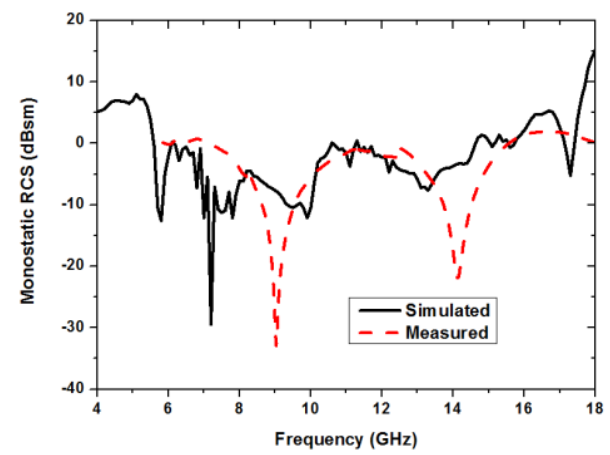

Fig. 18. Comparison between the simulated and measured monostatic RCS of the proposed checkerboard surface

\section{IV.CONCLUSION}

In this work, a bandwidth enhanced PRRS has been presented. Compared with the previously reported PRRS, the polarization rotation bandwidth of the proposed PRRS is enhanced from $49 \%$ to $97 \%$ with a high PCR of $96 \%$. The proposed PRRS was then applied to ultra-wideband RCS reduction. The PRRS is arranged in four orthogonal directions 
to form a checkerboard surface and the bandwidth of $10-\mathrm{dB}$ RCS reduction is over $103 \%$. The RCS reduction of the checkerboard surface is equal to the reflection coefficient $\mathrm{r}_{\mathrm{yy}}$ of the proposed PRRS. Simulated and experimental results illustrate the capability of the PRRS to reduce the RCS over an ultra-wideband and both the monostatic and bistatic RCS can be significantly reduced.

\section{REFERENCES}

[1] L. Ren, Y. Jiao, F. Li, J. Zhao, and G. Zhao, "A dual-layer T-shaped element for broadband circularly polarized reflectarray with linearly polarized feed," IEEE Antennas Wireless Propag. Lett., vol. 10, pp.407-410, 2011.

[2] M. Zhao, G. Zhang, X. Lei, J. Wu, and J. Shang. "Design of new single layer multiple-resonance broadband circularly polarized reflectarrays," IEEE Antennas Wireless Propag. Lett., vol. 12, pp.356-359, 2013.

[3] S. A. Muhammad, R. Sauleau, G. Valerio, L. L. Coq, and H. Legay, "Self polarizing Fabry-Perot antennas based on polarization twisting element," IEEE. Trans. Antennas Propag., vol. 61, no. 3, pp. 1032-1040, 2013.

[4] W. Yang, K.-W. Tam, W.-W. Choi, W. Che, and H. Hui, "Polarization rotation reflective surface based on artificial magnetic conductor and its application," Electron. Lett., vol. 50, no.21, pp. 1500-1502, 2015.

[5] M. Eular, V. Fusco, R. Cahill, and R. Dickie, "325 GHz single layer sub-millimeter wave FSS based split slot ring linear to circular polarization convertor," IEEE. Trans. Antennas Propag., vol. 58, no. 7, pp. 2457-2459, Jul. 2010

[6] J. Y. Chin, M. Lu, and T. J. Cui, "Metamaterials polarizers by electric-field-coupled resonators,"Appl. Phys. Lett., vol. 93, 251903, 2008.

[7] R. Singh, E. Plum, C. Menzel, C. Rockstuhl, A. K. Azad, R. A. Cheville, F. Lederer, W. Zhang, and N. I. Zheludev, "Terahertz metamaterial with asymmetric transmission," Phys. Rev. B, vol. 80, 153104, 2009.

[8] C. Menzel, C. Helgert, C. Rockstuhl, E.-B. Kley, A. Tunnermann, T. Pertsch, and F. Lederer, "Asymmetric Transmission of linearly polarized light at optical metamaterials," Phys. Rev. Lett., vol. 104, 253902, 2010.

[9] Y. Ye, and S. He, " $90^{\circ}$ polarization rotator using a bilayered chiral metamaterial with giant optical activity," Appl. Phys. Lett., vol. 96, 203501, 2010.

[10] Z. Wei, Y. Cao, Y. Fan, X. Yu, and H. Li, "Broadband polarization transformation via enhanced asymmetric transmission through arrays of twisted complementary split-ring resonators," Appl. Phys. Lett., vol. 99, 221907, 2011.

[11] H. Shi, A. Zhang, S. Zheng, J. Li, and Y. Jiang, "Dual-band polarization angle independent $90^{\circ}$ polarization rotator using twisted electric-field-coupled resonators," Appl. Phys. Lett., vol. 104, 034102, 2014.

[12] D. Yan, Q. Gao, C. Wang, C. Zhu, and N. Yuan, "A novel polarization convert surface based on artificial magnetic conductor," Proc. APMC2005, Suzhou, China, Dec. 2005

[13] X.-C. Zhu, W. Hong, K. Wu, H.-J. Tang, Z.-C. Hao, J.-X. Chen, and G.-Q. Yang, "A novel reflective surface with polarization rotation characteristic," IEEE. Trans. Antennas Propag., vol. 12, pp. 968-971, 2013.

[14] M. Feng, J. Wang, H. Ma, W. Mo, H. Ye and S. Qu, "Broadband polarization rotator based on multi-order plasmon resonances and high impedance surface," J. Appl. Phys., vol. 114, 074508, 2013.

[15] Y. Z. Cheng, W. Withayachumnankul, A. Upadhyay, D. Headland, Y. Nie, R. Z. Gong, M. Bhaskaran, S. Sriram, and D. Abbott, "Broadband terahertz reflective linear polarization convertor," Infrared, millimeter, and Terahertz waves, 2014 39th international conference on. IEEE, 2014.

[16] H. Chen, J. Wang, H. Ma, S. Qu, Z. Xu, A. Zhang, M. Yan, and Y. Li, "Ultra-wideband polarization conversion metasurfaces based on multiple plasmon resonances," J. Appl. Phys., vol. 115, 154504, 2014.

[17] X. Huang, D. Yang, and H. Yang, "Multiple-band reflective polarization converter using U-shaped metamaterial," J. Appl. Phys., vol. 115, 103505, 2014.

[18] W. Yang, K.-W. Tam, W.-W. Choi, W. Che, and H. T. Hui, "Novel polarization rotation technique based on an artificial magnetic conductor and its application in a low-profile circular polarization antenna," IEEE. Trans. Antennas Propag., vol. 62, no. 12, pp. 6206-6216, Dec. 2014.

[19] L. Zhang, P. Zhou, H. Lu, H. Chen, J. Xie, and L. Deng, "Ultra-thin reflective polarization rotator based on multiple plasmon resonances,"
IEEE Antennas Wireless Propag. Lett., 2015

[20] Y. Jia, Y. Liu, Y. J. Guo, K. Li, and S. Gong, "Broadband polarization rotation reflective surfaces and their application to RCS reduction,"

[21] M. Paquay, J.-C. Iriarte, I. Ederra, R. Gonzalo, and P. de Maagt, "Thin AMC structure for radar cross-section reduction," IEEE. Trans. Antennas Propag., vol. 55, no. 12, pp. 3630-3638, Dec. 2007.

[22] Y. Zhang, R. Mittra, B. Z. Wang, and N. T. Huang, "AMCs for ultra-thin and broadband RAM design," Electron. Lett., vol. 45, no. 10, pp. 484-485, 2009.

[23] J. C. I. Galarregui, A. T. Pereda, J. L. M. de Falcón, I. Ederra, R. Gonzalo, and P. de Maagt, "Broadband radar cross reduction using AMC technology," IEEE. Trans. Antennas Propag., vol. 61, no. 12, pp. 6136-6143, Dec. 2013.

[24] R.-B. Hwang, and Y.-L. Tsai, "Reflection characteristcs of a composite planar AMC surface," AIP Advances, vol. 2, 012128, 2012.

[25] H. T. Chen, J. Zhou, J. F. O'Hara, F. Chen, A. K. Azad, and A. J. Taylor, "Antireflection coating using metamaterials and identification of its mechanism," Phys. Rev. Lett., vol. 105, pp. 073901-1-073901-4, Aug. 2010

[26] H. T. Chen, "Interference theory of metamaterial perfect absorber," Opt. Expr., vol. 20, pp. 7165-7172, Mar, 2012.

[27] K. C. Hwang, "A novel meander-grooved polarization twist reflector," IEEE Microw. Wireless Compon. Lett., vol. 20, pp. 217-219, Apr. 2010

[28] H. Chen, H. Ma, S. Qu, J. Wang, Y. Li, H. Yuan, and Z. Xu, "Ultra-wideband polarization conversion metasurfaces," in Proc. IEEE APCAP, Jul. 2014, pp. 1009-1011.

[29] X. Gao, X. Han, W. Cao, H. Li, H. Ma, and T. J. Cui, "Ultra-wideband and high-efficiency linear polarization converter based on double V-shaped metasurface," IEEE. Trans. Antennas Propag., vol. 63, no. 8, pp. 3522-3530, Aug. 2015.

[30] E. F. Knott, J. F. Shaeffer, and M. T. Tuley, Radar Cross Section. Raleigh, NC, USA: SciTech Pub., 2004.

[31] W. Chen, C. A. Balanis, and C. R. Birtcher, "Checkerboard EBG surface for wideband radar cross section reduction," IEEE. Trans. Antennas Propag., vol. 63, no. 6, pp. 2636-2645, Jun. 2015.

[32] K. Wang, J. Zhao, Q. Cheng, D. S. Dong, T. J. Cui, "Broadband and broad-angle low-scattering metasurface based on hybrid optimization algorithm," Sci. Rep. 4, 5935 (2014).

[33] Y. Li, J. Zhang, S. Qu, J. Wang, H. Chen, Z. Xu, A. Zhang, "Wideband radar cross section reduction using two-dimensional phase gradient metasurfaces," Appl. Phys. Lett., vol. 104, 221110, 2014.

[34] A. Edalati, and K. Sarabandi, "Wideband, wide angle, polarization independent RCS reduction using nonabsorptive miniaturized-element frequency selective surfaces," IEEE. Trans. Antennas Propag., vol. 62, no. 2, pp. 747-754, Feb. 2014.

[35] ????and P. de Maagt, "Broadband radar cross reduction using AMC technology," IEEE. Trans. Antennas Propag., vol. 61, no. 12, pp. 6136-6143, Dec. 2013.

[36] R.-B. Hwang, and Y.-L. Tsai, "Reflection characteristcs of a composite planar AMC surface," AIP Advances, vol. 2, 012128, 2012

[37] Y. Li, J. Zhang, S. Qu, J. Wang, H. Chen, Z. Xu, A. Zhang, "Wideband radar cross section reduction using two-dimensional phase gradient metasurfaces," Appl. Phys. Lett., vol. 104, 221110, 2014.

[38] E. F. Knott, J. F. Shaeffer, and M. T. Tuley, Radar Cross Section. Raleigh, NC, USA: SciTech Pub., 2004. 